\title{
An Optimum Geometric Decoder for MIMO Systems
}

\author{
Z. Y. Shao, S. W. Cheung and T. I. Yuk \\ The University of Hong Kong, Hong Kong
}

\begin{abstract}
In this paper, we propose a simple implementation of an optimum Geometric decoder (GD) for use in MIMO systems. The decoder uses the channel matrix to construct a hyper paraboloid and then restricts the symbol vectors to be searched to a hyper ellipsoid projected from the hyper paraboloid. Computer simulation results on $4 \times 4$ different MIMO systems transmitting 16QAM and 64QAM show that the proposed GD can achieve the same bit-error-rate (BER) performances as those of the Maximum Likelihood (ML) decoder, yet having much less complexity.
\end{abstract}

Index Terms - MIMO systems, geometric decoding, maximum likelihood decoding, decoding complexity

\section{INTRODUCTION}

It is well-known that the channel capacity and errorrate performances of multiple-input multiple-output (MIMO) systems could be improved by increasing the numbers of transmit and receive antennas and sizes of constellation used for modulation $[1,2]$. However, to realize these advantages with optimum performances, Maximum-likelihood (ML) decoding employing exhaustive search is needed to be used at the receiver. The complexity of ML decoding increases exponentially with the numbers of transmit and receiver antennas and the sizes of constellation used. For large number of antennas with large constellation sizes, ML decoding will be impractical to implement.

Different low-complexity decoding algorithms such as zero forcing (ZF) and minimum-mean-square error (MMSE) linear equalizations (ZF, MMSE) and decision feedback equalization have been proposed for use in MIMO systems $[3,4]$, but these decoding algorithms are not optimum in terms of bit-error-rate (BER) performances. Sphere decoding (SD) has been introduced to significantly reduce the average decoding complexity [5], yet achieving the optimum performance as ML decoding. Both ML and SD decoding are search-

based algorithms. In ML decoding, the search is done for the whole lattice structure, while in SD, the search is restricted to a hyper sphere inside the lattice structure where the ML solution is likely to be inside the sphere. However, the decoding complexity for each received symbol vector very much depends on the initial setting for the radius of the hyper sphere. An inappropriate initial setting could lead to a huge complexity in decoding, so the choice of the initial radius is still an important problem to be dealt with in SD.
Using a geometric approach to decode the signals for the MIMO channels was first introduced in [6] and the technique was called Geometric decoding (GD) in [7]. GD is also a search-based algorithm. By observing the attributes of the channel matrix, GD searches the optimum lattice point on an ellipsoid paraboloid. Theoretically, for a complete search, GD could achieve the optimum performance as ML decoding, but then the complexity would be similar to that of ML decoder. In [7], a simplified GD algorithm was proposed for the cubic structure of pulse amplitude modulation (PAM) and quadrature amplitude modulation (QAM). The idea was to consider only the largest one or two eigenvalues and the corresponding eigenvectors to restrict the lattice points to be searched in a small subset. Since other lattice points were not considered in the search, it was not an optimum GD.

In this paper, we propose a simple but optimum GD algorithm implementation for use in the MIMO systems over block fading channels. The decoder constructs an elliptic paraboloid using the channel matrix and restricts the lattice points to be searched to a small region inside a hyper ellipsoid projected from the elliptic paraboloid using the zero forcing solution. A hyper rectangle enclosing the hyper ellipsoid is used to indentify the exact lattice points within hyper ellipsoid. Computer simulation has been used to assess the complexity reduction and performances of our proposed GD on $4 \times 4$ MIMO systems transmitting 16QAM and 64QAM over a block fading channel. Results show that our proposed GD is an optimum decoder and can achieve complexity reductions of more than $95 \%$ and $80 \%$ for 64QAM and 16QAM, respectively, over ML decoding.

The reminder of this paper is organized as follows. Section II describes the model of MIMO system used for studies. The general description on the concept of GD and our proposed GD implementation are presented in section III. An example illustrating the proposed GD is given in section IV. Simulation results and discussions are given in section V. Finally, conclusions are drawn in section VI.

\section{MIMO SYSTEM MODEL}

Consider an uncoded MIMO system with $N$ transmit and receive antennas over a block fading channel. The received signal matrix is given by:

$$
\mathbf{r}=\mathbf{H x}+\mathbf{w}
$$


where $\mathbf{r}=\left[r_{1}, r_{2}, \ldots, r_{N}\right]^{T}$ is the $N$-dimensional received signal vector, $\mathbf{x}=\left[x_{1}, x_{2}, \ldots, x_{N}\right]^{T}$ a possible symbol vector in the transmit lattice, with []$^{T}$ denoting vector or matrix transposition, $\mathbf{H}$ is a channel matrix, with elements $h_{i j}$ representing the transfer function from the $j$-th transmit antenna to $i$-th receive antenna, and $\mathbf{w}=\left[w_{1}, w_{2}, \ldots, w_{N}\right]^{T}$ is an independently and identically distributed (i.i.d.) zero-mean Gaussian noise vector with elements having a fixed variance. For real multi-level PAM (M-PAM) transmission, (1) has real-value elements and so is a real matrix equation. While for multi-level QAM (M-QAM) transmission, (1) has complex-value elements and but can be transformed into a real matrix equation:

$$
\left[\begin{array}{l}
\operatorname{Re}(\mathbf{r}) \\
\operatorname{Im}(\mathbf{r})
\end{array}\right]=\left[\begin{array}{cc}
\operatorname{Re}(H) & -\operatorname{Im}(H) \\
\operatorname{Im}(H) & \operatorname{Re}(H)
\end{array}\right]\left[\begin{array}{c}
\operatorname{Re}(\mathbf{x}) \\
\operatorname{Im}(\mathbf{x})
\end{array}\right]+\left[\begin{array}{c}
\operatorname{Re}(\mathbf{w}) \\
\operatorname{Im}(\mathbf{w})
\end{array}\right]
$$

with $\operatorname{Re}(\cdot)$ and $\operatorname{Im}(\cdot)$ being the real and imaginary parts, respectively, of $(\cdot)$. Since it is much easier and convenient to explain GD in the real dimensional space, without lost of generality, real-value vectors and matrices are assumed in (1) for discussions in the rest of the paper.

Assuming the channel matrix $\mathbf{H}$ in (1) is known at the receiver, a ML decoder calculates the Euclidean distances $\|\mathbf{r}-\mathbf{H x}\|^{2}$ between the received vector and all possible transmitted symbol vectors. The vector with the minimum Euclidean is detected as the transmitted vector symbol:

$$
\mathbf{x}_{\mathbf{M L}}=\arg \min \|\mathbf{r}-\mathbf{r} \in \mathbf{H}\|^{2}
$$

where $\mathbf{r}, \mathbf{x} \in \mathfrak{R}^{N}, \mathbf{H} \in \mathfrak{R}^{N \times N}$ and $\Omega$ is the set of all possible vector symbols in the transmitted lattice. For signals with a large number of lattice points, the computation requirement for ML decoding is too complicated to implement in practice. GD is one of the methods proposed to reduce the complexity.

\section{Proposed GeOMETRIC DECODING}

\section{A. Geometrical Analysis}

The Euclidean distance in (3) can be written as [7]:

$$
f(\mathbf{x})=\|\mathbf{r}-\mathbf{H} \mathbf{x}\|^{2}
$$

$$
\begin{aligned}
& =\left(\mathbf{x}-\mathbf{x}_{\mathbf{c}}\right)^{\mathbf{T}} \mathbf{H}^{\mathrm{T}} \mathbf{H}\left(\mathbf{x}-\mathbf{x}_{\mathbf{c}}\right) \\
& =\left(\mathbf{x}-\mathbf{x}_{\mathbf{c}}\right)^{\mathbf{T}} \mathbf{M}^{-1}\left(\mathbf{x}-\mathbf{x}_{\mathbf{c}}\right)
\end{aligned}
$$

which is an elliptic paraboloid in a $N+1$ dimensional space with the axis perpendicular to a subspace spanned by the symbol vectors in $\Omega$ and having a global minimum point given by $\mathbf{x}_{\mathbf{c}}=\mathbf{H}^{-1} \mathbf{r}$ as shown in Fig. 1 $[9,10]$.

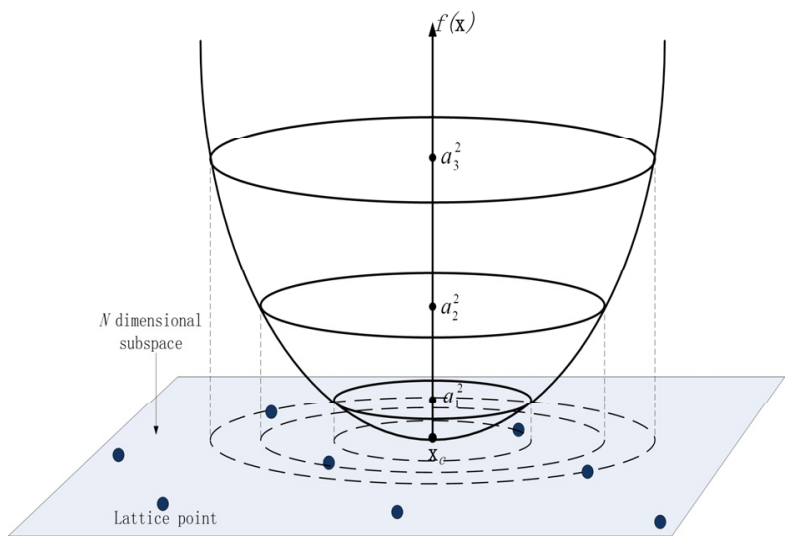

Fig. 1 Elliptic paraboloid in an $N+1$ dimensional space with axis perpendicular to a subspace spanned by the vectors in $\Omega$

Using eigenvalue decomposition, the matrix $\mathbf{M}$ in (5) can be written as:

$$
\mathbf{M}=\left(\mathbf{H}^{\mathrm{T}} \mathbf{H}\right)^{-1}=\left(\mathbf{V} \Lambda \mathbf{V}^{T}\right)^{\mathbf{T}}
$$

where $\Lambda=\operatorname{diag}\left(\lambda_{1}, \lambda_{2}, \ldots, \lambda_{N}\right)$ is the eigenvalues of $\mathbf{M}$ in descending order, and $\mathbf{V}$ is the corresponding eigenvector matrix given by:

$$
\begin{aligned}
\mathbf{V} & =\left[\mathbf{v}_{1}, \mathbf{v}_{2}, \mathbf{v}_{\mathbf{3}}, \cdots, \mathbf{v}_{\mathbf{N}}\right] \\
& =\left[\begin{array}{ccccc}
v_{11} & v_{21} & v_{31} & \cdots & v_{N 1} \\
v_{12} & v_{22} & v_{32} & \cdots & v_{N 2} \\
v_{13} & v_{23} & v_{33} & \cdots & v_{N 3} \\
\vdots & \vdots & \vdots & \ddots & \vdots \\
v_{1 n} & v_{2 n} & v_{3 n} & \cdots & v_{N N}
\end{array}\right]
\end{aligned}
$$

The horizontal cross section of the elliptic paraboloid (5) given by:

$$
f(\mathbf{x})=a^{2}
$$

which is a hyper ellipsoid with its center at a distance $a^{2}$, where $a \in \mathfrak{R}$, from the global minimum point $\mathbf{x}_{\mathbf{c}}$ This hyper ellipsoid, with lengths and directions of the 
semiaxes given by $a \sqrt{\lambda_{i}}$ and $\mathbf{v}_{\mathbf{i}}$, respectively [7], can be projected on to the subspace spanned by the vectors in $\Omega$ as shown in Fig. 1. Different values of $a^{2}$ in (9) produce different hyper ellipsoids on the subspace. All the transmitted lattice points must be lying on the elliptic paraboloid (5) and so a set of hyper ellipsoids containing the entire lattice can be obtained and projected on the subspace. The optimum GD solution is:

$$
\mathbf{x}_{\mathbf{G D}}=\arg \min _{\mathbf{x} \in \Omega} f(\mathbf{x}) .
$$

Thus the symbol vector lying on the hyper ellipsoid with the smallest value of $a^{2}$ (i.e. the smallest hyper ellipsoid) is decoded as the transmitted symbol vector. Since (10) is derived directly from (3), GD has the same performance as ML decoding and is optimum. Apparently, if the entire lattice points are considered by (10) for obtaining the decoding solution, it will have the same implementation complexity as that of the ML decoder. Simpler implementations have been proposed [7], but the performances were only suboptimum. Here we propose a simple, novel and optimum GD searching algorithm based on an integral hyper ellipsoid having the same dimension as that of the original lattice point space.

\section{B. Proposed Geometric decoding (GD) algorithm}

Our proposed implementation of GD consists of three steps described as follows.

\section{Step 1: Using zero-forcing solution to obtain a possible} hyper ellipsoid

We use zero-forcing decoding to obtain the initial solution $\mathbf{x}_{z f}$ [10], compute $a_{z f}{ }^{2}$ using (5) and then obtain a hyper ellipsoid with:

$$
f(\mathbf{x})=a_{z f}^{2}
$$

on the subspace spanned by the symbol vector in $\Omega$. The lattice points to be searched in GD are limited to be inside this hyper ellipsoid, instead of the whole the subspace, thus the complexity could be significantly reduced. Since zero-forcing is not optimum decoding process, the optimum solution must be inside this hyper ellipsoid.

\section{Step 2: Making use of circumscribed hyper rectangle}

It is very complicated to find all the symbol vectors inside the hyper ellipsoid using (11), because in general the semiaxes are not in parallel with the axes of the lattice point space. Here we construct a smallest hyper rectangle which encloses the hyper ellipsoid. We use a new $N$-dimensional rectangular coordinate system, with $x_{i}^{\prime}$ - axes, for $i=1,2,3, \cdots, N$, coinciding with the $i^{\text {th }}$ - semiaxis of the hyper ellipsoid and the origin coinciding with $\mathbf{x}_{\mathbf{c}}$. The superscript prime is used to denote the variables in the new coordinate system. The hyper rectangle in an $N$-dimensional space will have $2^{N}$ apexes. The coordinates of these apexes are composed of $N$ variables in these $x_{i}^{\prime}$ - axes directions and can be denoted in the new coordinate system as:

$$
\mathbf{k}_{\mathbf{p}}{ }^{\prime}=\left[x_{p 1}^{\prime}, x_{p 2}^{\prime}, x_{p 3}^{\prime}, \cdots, x_{p N}^{\prime}\right]
$$

where $p=1,2, \cdots, 2^{N}$ and $x_{p i}^{\prime}= \pm a_{z f} \sqrt{\lambda_{i}}$, for $i=1,2, \ldots$, $N$. By using coordinate transformation, it can easily be shown that the coordinates of these $2^{N}$ apexes can be transformed to the lattice point space as:

$$
\mathbf{k}_{\mathbf{p}}^{\mathrm{T}}=\mathbf{V} \cdot\left(\mathbf{k}_{\mathbf{p}}\right)^{\mathrm{T}}+\mathbf{x}_{\mathbf{c}}
$$

where $\mathbf{V}$ is the eigenvector matrix (7). Thus the $i^{\text {th }}$ component in $\mathbf{k}_{\mathbf{p}}$ can be obtained as:

$$
x_{p i}=\sum_{q=1}^{N}\left(v_{q i} x_{p q}^{\prime}\right)+x_{c i}
$$

where $x_{c i}$ is the $i^{\text {th }}$-component in $\mathbf{x}_{\mathbf{c}}$ in the $x_{i}$-axis direction. The perimeters of this smallest hyper rectangle are not in parallel with the axes of the lattice point space, so it is not convenient to use. We need to find another hyper rectangle which encloses this smallest hyper rectangle and has the perimeters in parallel with the axes of the lattice point space.

Since $x_{p q}^{\prime}= \pm a_{z f} \sqrt{\lambda_{i}}$, the maximum and minimum values of the element in $\mathbf{k}_{\mathbf{p}}$ in the $x_{i}$ - axis direction of the lattice space is:

$$
\begin{aligned}
& x_{i_{-} \text {max }}=x_{c i}+\sum_{q=1}^{N}\left|v_{q i} a_{z f} \sqrt{\lambda_{q}}\right| \\
& x_{i_{-} \text {min }}=x_{c i}-\sum_{q=1}^{N}\left|v_{q i} a_{z f} \sqrt{\lambda_{q}}\right|
\end{aligned}
$$

The new hyper rectangle enclosing the previous hyper rectangle has the apexes with coordinates given by:

$$
\hat{\mathbf{k}}_{\mathbf{p}}=\left[\breve{x}_{p 1}, \breve{x}_{p 2}, \breve{x}_{p 3}, \cdots, \breve{x}_{p N}\right]
$$

where $p=1,2, \cdots, 2^{N}$ and $\breve{x}_{p i}=x_{i_{-} \max }$ or $x_{i_{-} \min }$, for $i=1$, $2, . ., N$. Assume that, within the boundaries of $x_{i_{\text {m }} \max }$ and $x_{i_{-} \min }$ given by (15), there are iNum symbol vectors, with coordinates in the $x_{i}$ - axis direction given by: 


$$
\left\{\hat{x}_{i}\right\}=\left\{\hat{x}_{i 1}, \hat{x}_{i 2}, \hat{x}_{i 3}, \cdots \hat{x}_{i i N u m}\right\}
$$

If $i N u m=0$, there is no symbol vector inside the hyper rectangle and the GD needs to do no searching. The zero-forcing point is considered to be the solution. If $i$ Num is not zero for $i=1, \ldots, N$, the total number of symbol vectors inside the hyper rectangle is $\prod_{i=1}^{N} i N u m$ which must include all the symbol vectors inside the enclosed hyper ellipsoid. Then we narrow the search region to be exactly inside the hyper ellipsoid as described in Step 3.

\section{Step 3 Narrowing search region to hyper ellipsoid}

Assume that among all possible value sets $\left\{\hat{x}_{i}\right\}$ of (17), for $i=1,2, \ldots, N$, the set $\left\{\hat{x}_{l}\right\}$ in the $l^{t h}$-axis direction, where $1 \leq l \leq N$, has the largest number of elements. The rest of the value sets can be used to form $\prod_{i=1, i \neq l}^{N} i N u m$ vectors with different combinations given by:

$\mathbf{c}_{\mathbf{k}}=\left[\hat{x}_{1}\left(k_{1}\right), \hat{x}_{2}\left(k_{2}\right), \cdots \hat{x}_{l-1}\left(k_{l-1}\right), \quad, \hat{x}_{l+1}\left(k_{l+1}\right), \cdots, \hat{x}_{N}\left(k_{N}\right)\right]$

where $k=1,2, \ldots, \prod_{i=1, i \neq l}^{N} i N u m$ and the $i^{\text {th }}$-component in $\mathbf{c}_{\mathbf{k}}$ is taken from the $k_{j}^{\text {th }}$-component in the value set $\left\{\hat{x}_{i}\right\}$ given by (17), with $k_{j}=1,2, \ldots, j N u m$ (that is $k_{1}=1,2, \ldots, 1 \mathrm{Num} \quad, \quad k_{2}=1,2, \ldots, 2 \mathrm{Num} \quad$, $k_{3}=1,2, \ldots, 3 \mathrm{Num}$ and so on). The elements in $\mathbf{c}_{\mathbf{k}}$ are substituted into (11) to find the $l^{\text {th }}$ coordinates of the symbol vectors within the hyper ellipsoid, i.e.:

$$
\left\{\bar{x}_{l}\right\}=\left\{\bar{x}_{l 1}, \bar{x}_{l 2}, \bar{x}_{13}, \cdots \bar{x}_{\text {lNum }}\right\}
$$

where lNum is the number of symbol vectors. Each of the elements in (19) is used in (18) to form a complete set of coordinates for a symbol vector:

$$
\mathbf{x}_{\mathbf{k}}=\left[\hat{x}_{1}\left(k_{1}\right), \hat{x}_{2}\left(k_{2}\right), \cdots \hat{x}_{l-1}\left(k_{l-1}\right), \bar{x}_{l}\left(k_{l}\right), \hat{x}_{l+1}\left(k_{l+1}\right), \cdots, \hat{x}_{N}\left(k_{N}\right)\right]^{T}
$$

inside the hyper ellipsoid. Finally, the value of $a^{2}$ for each symbol vector is computed using (9) and the one with minimum $a^{2}$ is decoded as the transmitted symbol vector.

\section{EXAMPLE}

Consider a $2 \times 2$ 8-PAM MIMO system with a 2dimensional lattice set. Assumed that, at the receiver, the ellipse has been determined using our proposed zero forcing method and is shown in Fig. 2, where the global minimum point is given by $\mathbf{x}_{\mathbf{c}}=\mathbf{H}^{-1} \mathbf{r}$. The semiaxes of the ellipse has lengths of $a_{z f} \sqrt{\lambda_{1}}$ and $a_{z f} \sqrt{\lambda_{2}}$ with respective directions of $\mathbf{v}_{\mathbf{1}}$ and $\mathbf{v}_{\mathbf{2}}$ given by (9). The smallest rectangle enclosing the ellipse has four apexes, $A, B, C$ and $D$, with the coordinates in the new rectangular coordinate system given by:

$$
\begin{aligned}
& A=\left(-a_{z f} \sqrt{\lambda_{1}},-a_{z f} \sqrt{\lambda_{2}}\right), B=\left(-a_{z f} \sqrt{\lambda_{1}},+a_{z f} \sqrt{\lambda_{2}}\right), \\
& C=\left(a_{z f} \sqrt{\lambda_{1}},-a_{z f} \sqrt{\lambda_{2}}\right) \text { and } D=\left(a_{z f} \sqrt{\lambda_{1}},+a_{z f} \sqrt{\lambda_{2}}\right),
\end{aligned}
$$

Substituting these vectors into (14) yields the corresponding coordinates in the lattice point space. It can be seen from Fig. 2 that the maximum and minimum $x_{1}$-coordinate values are given by points $A$ and $D$ as $x_{1 \_ \text {min }}$ and $x_{1 \_ \text {max }}$, respectively. Within the boundaries of $x_{1_{1} \min }$ and $x_{1 \_ \text {max }}$, there are two possible lattice points having the set $\left\{\hat{x}_{1}\right\}=\left\{\hat{x}_{11}, \hat{x}_{12}\right\}=\{1,3\}$ as expressed in (17) for the $x_{1}$-axis coordinate. While in the $x_{2}$-axis direction, the maximum and minimum values are given by points $B$ and $C$ as $x_{2_{2} \min }$ and $x_{2_{-} \max }$, respectively, and within which there is only one possible lattice point, i.e., set $\left\{\hat{x}_{2}\right\}=\left\{\hat{x}_{21}\right\}=\{1\}$. Since the number of elements in $\left\{\hat{x}_{1}\right\}$ is larger than the number of element in $\left\{\hat{x}_{2}\right\}$, we substitute the element in $\mathbf{c}_{1}=\left\{\hat{x}_{21}\right\}=\{1\}$ into the hyper ellipsoid equation (11) to obtain the corresponding values of $\bar{x}_{1 \max }^{1}$ and $\bar{x}_{1 \min }^{1}$. It can be seen in Fig. 2 that the only possible lattice point between $\bar{x}_{1 \max }^{1}$ and $\bar{x}_{1 \min }^{1}$ has the $x_{1}$-axis coordinate of $\left\{\bar{x}_{1}\right\}=\left\{\bar{x}_{11}\right\}=\{3\}$, as indicated as point $\mathbf{x}_{1}$ with coordinate of $\left[\begin{array}{ll}3 & 1\end{array}\right]^{T}$. This is the only point inside the ellipse and so is the solution.

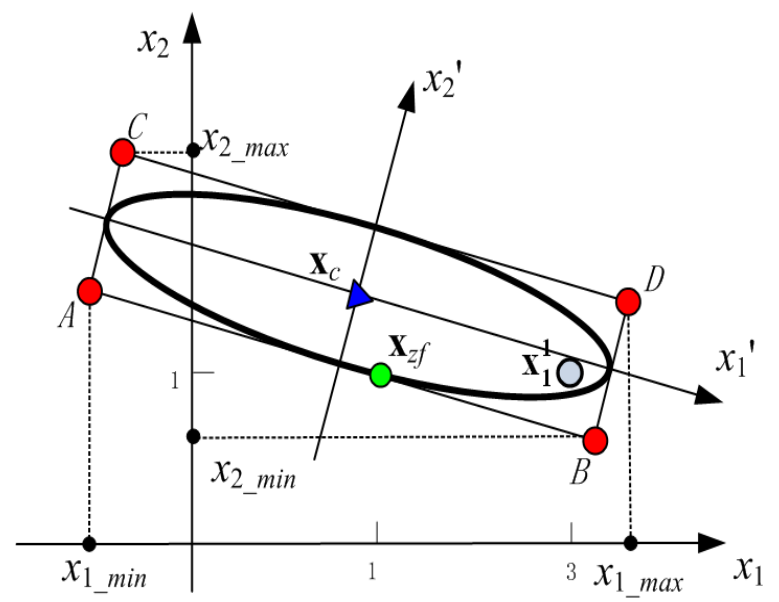

Fig.2. Proposed GD algorithm for a $2 \times 2$ 8-PAM MIMO system 


\section{SimUlation RESUlTS AND DisCUSSIONS}

Matlab simulation has also been used to assess the performances of our proposed GD and ML used in the $4 \times 4$ MIMO systems transmitting 16QAM and 64QAM in a block-fading channel. Results on the BERs of these systems are shown in Fig. 3. It can be seen that our proposed GD has the same performance as the ML decoder, indicating that our proposed GD is optimum. This is expected because both ML decoder and our proposed GD attempt to find the same symbol vector with the minimum distance and decode it as the transmitted symbol vector. However, our proposed GD can speed up the decoding process by narrowing the search region and this does not affect the search result.

The complexity reduction of our proposed GD here is measured by the simulation time required. Table 1 shows the ratios (in percentages) of the simulation time required by GD to that required by $\mathrm{ML}$ decoding in these MIMO systems at the signal-to-noise ratios (SNRs) of 15, 20 and $25 \mathrm{~dB}$. It can be seen that GD requires substantially less simulation times than $\mathrm{ML}$ decoding in these two MIMO systems in all tests. For $64 Q A M$, the GD requires only less than $5 \%$ the time required by using ML decoding in all tests, i.e., reducing the complexity by more than $95 \%$. For 16QAM, the reduction is more than $85 \%$. These are expected because, for ML decoding, all the lattice points are used, so the implementation complexity increases exponentially with the number of antennas and the modulation levels used in the MIMO systems. While for $\mathrm{GD}$, the lattice points are limited to those inside the hyper ellipsoid that was generated using zero forcing. It also can be seen that the reduction is more for 64QAM than for 16QAM and also more at higher SNRs.

TABLE I

RATIOS OF SIMULATION TIMES TAKEN BY GD TO THAT TAKEN BY ML

\begin{tabular}{lccc}
\hline \hline & $\mathbf{1 5} \mathbf{~ d B}$ & $\mathbf{2 0} \mathbf{d B}$ & $\mathbf{2 5} \mathbf{d B}$ \\
\hline 16QAM & $14.67 \%$ & $12.67 \%$ & $3.49 \%$ \\
64QAM & $4.35 \%$ & $2.26 \%$ & $2.56 \%$ \\
\hline \hline
\end{tabular}

\section{CONCLUSIONS}

A simple implementation of the GD algorithm for optimum decoding of signals for MIMO systems has been presented. The decoder uses the channel matrix and the zero forcing solution to construct a hyper paraboloid containing all the transmit symbol vectors. The GD restricts the symbol vectors to be searched to those inside the hyper ellipsoids projected from the hyper paraboloid. Computer simulation results for MIMO systems transmitting 16QAM and 64QAM show that the proposed GD can achieve complexity reduction of more than $85 \%$ and $95 \%$, respectively, over the ML decoder, yet having the same optimum BER performances.

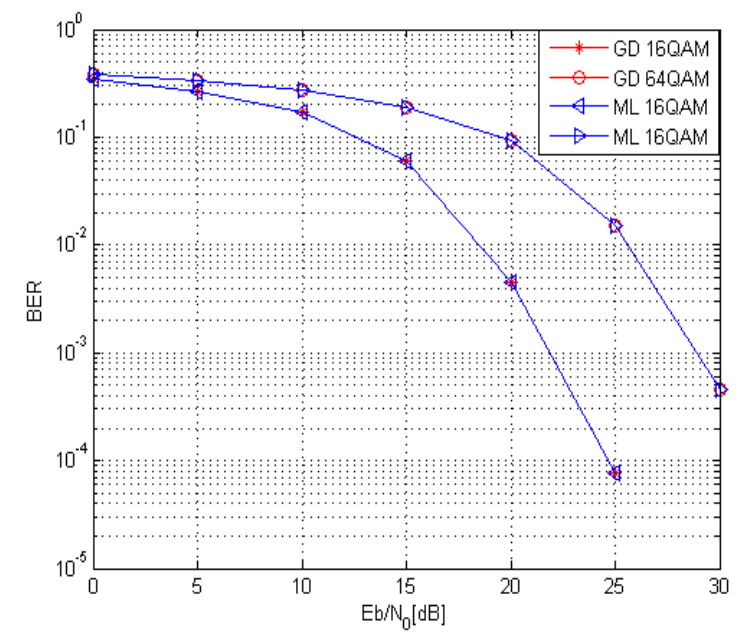

Fig.3. BERs of $4 \times 4$ 16QAM and 64QAM using proposed GD and ML in block-fading channel.

\section{REFERENCES}

[1] A. Goldsmith, S. A. Jafar, N. Jindal and S. Vishwanath, "Capacity limits of MIMO channels," IEEE Journal on Selected areas in Communications, vol. 21, no. 5, pp. 684-702, Jun. 2003.

[2] G. J. Foschini and M. J. Gans, "On limits of wireless communications in a fading environment when using multiple antennas," Wireless Personal Commun., vol. 6, pp. 311-335, Mar. 1998.

[3] P. Wolniansky, G. J. Foschini, G. Golden, and R. Valenzuela, "V-BLAST: an architecture for realizing very high data rates over the rich-scattering wireless channel," in International Symposium on Signals, Systems and Electronics ISSSE98, pp. 295-300.

[4] G. J. Foschini, "Layered space-time architeture for wireless communications in a fading environment when using multi-element antennas," Bell labs Tech. j., vol. 2, pp. 41-59, 1996.

[5] E. Viterbo and J. Boutros, "A Universal Lattice Code Decoder for Fading Channels," IEEE Trans. Information Theory, vol. 45, no. 5, pp. 1639-1642, July 1999.

[6] H. Artes, D. Seethaler, and F. Hlawatsch, "Efficient detection algorithms for mimo channels: A geometrical approach to approximate $\mathrm{ml}$ detection," IEEE Trans. Signal Processing, vol. 51, no. 11, pp. 2808-2820, Nov. 2003.

[7] M. Samuel and M. P. Fitz, "Geometric Decoding Of PAM and QAM Lattices," in Proc. IEEE Global Telecommunications Conf., pp. 4247-4252, Nov.2007.

[8] S. Boyd and L. Vandenberghe, Convex Optimization. Cambridge University Press, 2004.

[9] R. A. Horn and C. R. Johnson, Matrix Analysis. Cambridge University Press, 1999.

[10] A. Paulraj, R. Nabar and D. Gore, Introduction to SpaceTime Wireless Communications. Cambridge University Press, May 2003. 